\title{
Estudio, caracterización y optimización del diseño de redes DWDM
}

\section{Characterization and optimization of DWDM network design}

\author{
Ilber Adonayt Ruge Ruge \\ Ingeniero electrónico, magíster en Ingeniería de Control Industrial. Docente de \\ la Universidad de Cundinamarca. Fusagasugá, Colombia. \\ Contacto:iruge@mail.unicundi.edu.co \\ Humberto Numpaque López \\ Ingeniero electrónico, magíster en Ingeniería de Control Industrial. Docente de \\ la Universidad de Cundinamarca. Fusagasugá, Colombia. \\ Contacto:ihnump@gmail.com \\ Yelidza Nayive Medina Moyano \\ Ingeniera electrónica, candidata a especialista en Seguridad Informática. Univer- \\ sidad de Cundinamarca. Fusagasugá, Colombia. \\ Contacto: niyimedina@gmail.com \\ Ingrid Carolina Ortiz Álvarez \\ Ingeniera electrónica, candidata a especialista en Seguridad Informática. Univer- \\ sidad de Cundinamarca. Fusagasugá, Colombia. \\ Contacto: caroortiz0627@hotmail.com
}

Fecha de recepción: 21 de noviembre de 2011

Clasificación del artículo: Investigación

Fecha de aceptación: 28 de agosto de 2012

Financiamiento: Universidad de Cundinamarca

Palabras clave: algoritmos genéticos, diseño, DWDM, enlace, optimización, red.

Key words: genetic algorithms, design, DWDM, link, optimization, net. 


\section{RESUMEN}

Este proyecto describe el diseño de una red DWDM aplicando la técnica de optimización de algoritmos genéticos, cuyo objetivo principal es determinar la mínima cantidad de amplificadores ópticos necesarios para operar una red y la ubicación exacta de ellos en cada enlace de la red. Se optimiza el número de amplificadores ópticos debido a que estos componentes son los más costosos y sus precios varían entre el rango de 20.000 a 60.000 euros dependiendo de su potencia.

\section{ABSTRACT}

This project describes the DWDM network design using genetic-algorithm optimization techniques. The main purpose is to determine the minimum amount of optical amplifiers needed to operate a network as well as the exact location of each network link. The number of optical amplifiers is optimized since these components are extremely costly; their prices vary in the range of 20.000 to 60.000 euros depending on their power.

\section{INTRODUCCIÓN}

DWDM es una técnica para insertar información de diferentes estaciones de trabajo a una fibra óptica. Generalmente, esta técnica es empleada para diseñar redes ópticas de área local, metropolitana y amplia (LAN, MAN y WAN) permitiendo tener un ancho de banda de aproximadamente $140 \mathrm{THz}$ en la banda de 900 a $1600 \mathrm{~nm}$, estas redes soportan aplicaciones de video, voz y datos con velocidades de transmisión de 10Tbits/s y tasas de error de bit (BER) del orden de con respecto a los sistemas de comunicaciones convencionales [1].

El diseño de redes ópticas se basa en presupuestos de potencia de cada enlace que conforman la red, en estos presupuestos se consideran las pérdidas por efecto de atenuación en la fibra óptica y los diferentes dispositivos como: fibras ópticas, transmisores ópticos (laser y led), receptores ópticos (PIN y APD), acopladores ópticos tipo estrella pasivos, amplificadores ópticos, filtros ópticos, multiplexores y demultiplexores ópticos, además de las limitaciones en potencia de cada uno de ellos [2]. Para reducir el efecto de atenuación de la señal, conforme esta se propaga en la red, se emplea amplificación de naturaleza eléctrica o óptica, sin embargo, la amplificación eléctrica hace que el sistema pierda velocidad de transmisión ya que es necesario convertir la señal de naturaleza óptica a eléctrica, para poderla amplificar y posteriormente llevarla al dominio óptico. Para evitar esta problemática se han desarrollado amplificadores ópticos tipo RAMMAN, EDFA y SOA, la desventaja de emplear estos dispositivos es que, comercialmente, son costosos, de esta manera el diseño de redes ópticas se convierte en un problema desafiante, debido a que se debe garantizar determinado nivel de potencia en cada uno de los enlaces, empleando la mínima cantidad de amplificadores ópticos, de esta forma se asegura que la señal llegará a cada uno de los receptores con los niveles mínimos de potencia para que estos la puedan detectar coherentemente [3].

Este articulo muestra la metodología de diseño de redes de área metropolitana empleando la técnica DWDM y optimizando el número de amplificadores ópticos requeridos en la red mediante el uso de algoritmos genéticos 


\section{investigación}

\section{METODOLOGÍA}

\subsection{Diseño de una red DWDM}

A continuación, se presenta la metodología para el diseño de redes de área metropolitana empleando la técnica de acceso al medio DWDM, este diseño se hace por medio de cuatro módulos que se explicarán más adelante.

Para este diseño, se escogió una red que ya estuviera implementada, con el fin de obtener distancias reales y un trayecto ya establecido, por esto se eligió el trayecto Barranquilla - Manizales que tiene una distancia de $825 \mathrm{Km}$.

Se escogió esta trayectoria ya que los sitios que la componen cuentan con facilidades de instalación, mantenimiento $\mathrm{y}$, además, cumple con la energía suficiente para la alimentación de los dispositivos de la red. Por consiguiente, la red DWDM que se va a diseñar utiliza una topología malla, la cual está conformada por la topología punto a punto y anillo, permitiendo de esta manera que, si algún enlace (fibra óptica) se rompe o se daña, se pueda tomar otra trayectoria para llegar a su destino sin perder información. Tendrá como objetivo minimizar el número de amplificadores por medio de la técnica de optimización de algoritmos genéticos, además se realizará una comparación con las ecuaciones enteras lineales.

En la figura 1 se observa la red de área metropolitana DWDM en donde las estrellas ópticas pasivas se representan mediante un círculo, los grupos de estaciones se representan por medio de cuadros, los enlaces de subida son las líneas rojas y los de bajada las líneas azules.

Los datos que se requieren para el diseño de esta red se encuentran en la tabla 2.

Este diseño [4] requiere los datos técnicos de los dispositivos utilizados en la red, los cuales son: máxima potencia del transmisor en cada una de
Tabla 1. Enlaces a usar en el diseño propuesto.

\begin{tabular}{|l|c|}
\hline Localización geográfica & $\begin{array}{c}\text { Distancia } \\
\text { (Km) }\end{array}$ \\
\hline Barranquilla - Carmen de Bolívar & 151,2 \\
\hline Carmen de Bolívar - Sincelejo & 61,6 \\
\hline Sincelejo - Planeta Rica & 112,1 \\
\hline Planeta Rica - Caucacia & 67,2 \\
\hline Caucacia - Yarumal & 138,1 \\
\hline Yarumal - Itagüí & 109 \\
\hline Itagüí - Caramanta & 74,4 \\
\hline Caramanta - Manizales & 111,4 \\
\hline TOTAL & 825 \\
\hline
\end{tabular}

Fuente: elaboración propia

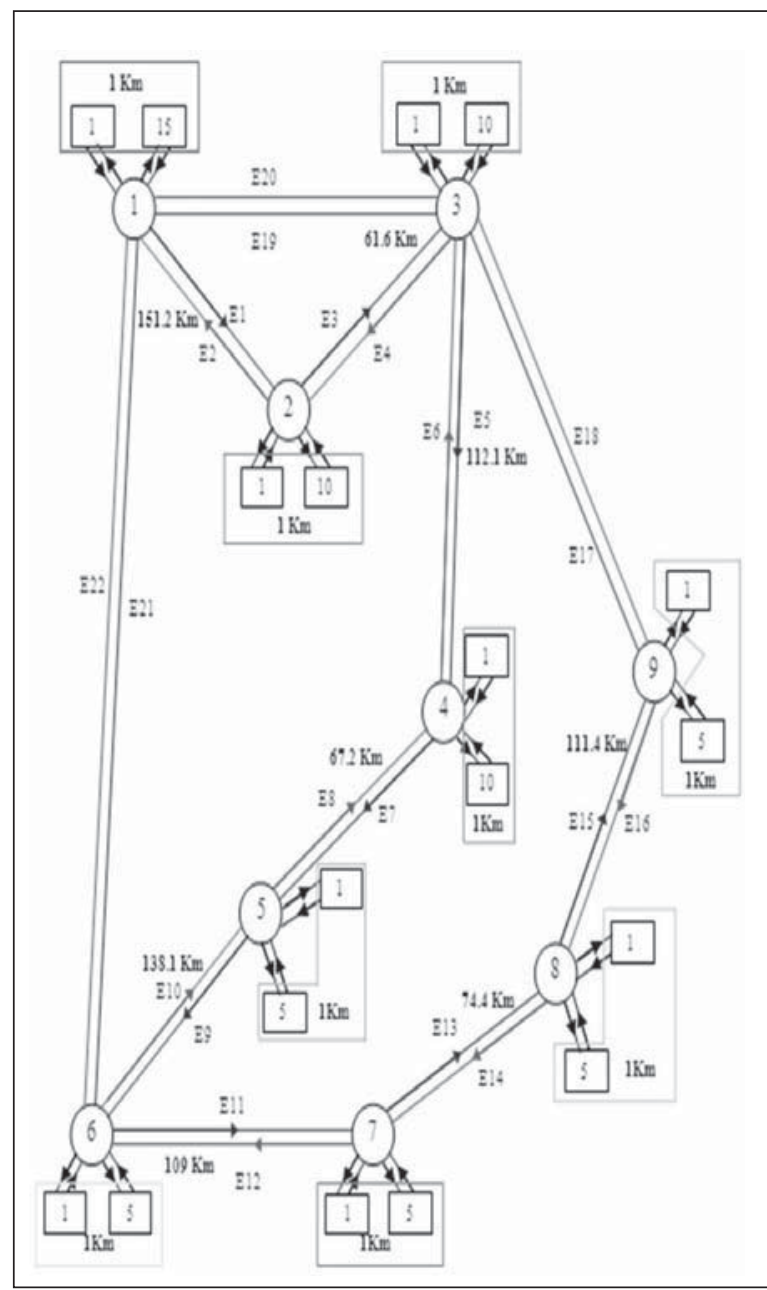

Figura 1. Red de área metropolitana DWDM.

Fuente: elaboración propia 
las estaciones, $\mathrm{P}_{\max }=3 \mathrm{~dB}$; mínima potencia a la entrada del receptor en cada una de las estaciones para que la señal sea detectada, $P_{\text {sen }}=-32 \mathrm{dBm}$; constante de atenuación en la fibra, $\alpha=0.25 \mathrm{~dB} /$ $\mathrm{Km}$; potencia mínima de señal a la entrada del amplificador, $\mathrm{P}_{\text {sen }}=-32 \mathrm{dBm}$; potencia de salida del amplificador, $\mathrm{P}_{\max }=0 \mathrm{~dB}$; margen de diseño, $\mathrm{MD}=3 \mathrm{~dB}$; longitud del enlace. También se requieren de los datos del diseño que son: número de estrella pasivas ópticas en la red, $\mathrm{M}=9$; número de estaciones de acceso en la red, $\mathrm{N}=70$; número de enlaces en la red (incluyendo enlaces de acceso y enlaces entre las estrellas), $\mathrm{L}=156$.

Para el diseño de esta red, se parte de cuatro módulos los cuales se explicaran a continuación: el primer modulo es la prueba de viabilidad, que determina si la red es viable para optimizar el número de amplificadores en la red y su ubicación en esta. Para esto utiliza la ecuación (1):

$$
\begin{gathered}
P_{\text {sen }} \leq P_{\text {max }}-10 \log \left(D_{i}-1\right)-10 \log \left(\lambda_{j}\right) \leq 3- \\
10 \log (16-1)-10 \log (65) \leq-26,89 \mathrm{dBm}
\end{gathered}
$$

Para esta ecuación y las demás que se encuentran en cada uno de los módulos se utilizan los datos que se encuentran en la tabla 2, en este caso son: la estrella que tiene el mayor grado de la estrella y la mayor cantidad de longitudes de onda que entran a la estrella. En este caso la red es viable, ya que el valor de $\mathrm{P}_{\max }$ es menor a $\mathrm{P}_{\text {sen. }}$.

El segundo módulo es la generación de restricciones para los enlaces, éste tiene como restricciones los enlaces agregados que tienen cada estrella hacia los grupos de estaciones y viceversa. También se encuentran las restricciones para los enlaces y para las estrellas. A continuación se mostrará el enlace entre estrellas. Para el enlace uno, ecuaciones (2), (3) y (4):

\begin{tabular}{|c|c|c|c|c|c|}
\hline $\begin{array}{l}\text { Número de } \\
\text { enlaces }\end{array}$ & $\begin{array}{l}\text { De qué estrella } \\
\text { viene y a cuál } \\
\text { se dirige }\end{array}$ & $\begin{array}{c}\text { Distancias } \\
\text { entre estrellas } \\
(\mathrm{Km})\end{array}$ & $\begin{array}{l}\text { Número } \\
\text { de longitudes } \\
\text { de onda }\end{array}$ & $\begin{array}{c}\text { Di grado } \\
\text { de la estrella }\end{array}$ & $\begin{array}{l}\text { Número de longitudes } \\
\text { máximas que entran } \\
\text { a cada estrella }\end{array}$ \\
\hline 1 & $1-2$ & 151,2 & 15 & 16 & 55 \\
\hline 2 & $2-1$ & 151,2 & 55 & 12 & 45 \\
\hline 3 & $2-3$ & 61,6 & 25 & 12 & 35 \\
\hline 4 & $3-2$ & 61,6 & 45 & 12 & 35 \\
\hline 5 & 3-4 & 112,1 & 35 & 7 & 45 \\
\hline 6 & $4-3$ & 112,1 & 35 & 7 & 50 \\
\hline 7 & $4-5$ & 67,2 & 45 & 7 & 55 \\
\hline 8 & $5-4$ & 67,2 & 25 & 7 & 60 \\
\hline 9 & $5-6$ & 138,1 & 50 & 6 & 65 \\
\hline 10 & $6-5$ & 138,1 & 20 & & \\
\hline 11 & $6-7$ & 109 & 55 & & \\
\hline 12 & $7-6$ & 109 & 15 & & \\
\hline 13 & $7-8$ & 74,4 & 60 & & \\
\hline 14 & 8-7 & 74,4 & 10 & & \\
\hline 15 & $8-9$ & 111,4 & 65 & & \\
\hline 16 & $9-8$ & 111,4 & 5 & & \\
\hline
\end{tabular}

Tabla 2. Datos de las características de la Red DWDM.

Fuente: elaboración propia 


$$
\begin{gathered}
P_{1}=P_{2}-\propto * L_{1}-10 \log \left(D_{1}-1\right)+S G_{1} \\
P_{1}=P_{2}-0,25 * 151,2 K m-10 \log (16-1)+S G_{1} \\
P_{1}=P_{2}-49,56+S G_{1}
\end{gathered}
$$

Como se desea compensar las pérdidas en el enlace uno utilizando el menor número de amplificadores ecuación (5), se requiere que:

$$
g_{\max _{1}} *\left(n_{1}-1\right)<S G_{1} \leq g_{\max _{1}} * n_{1}
$$

Donde, ecuaciones (6), (7) y (8).

$$
\begin{gathered}
g_{\text {max }_{1}}=\left|P_{\text {sen }}\right|-10 \log \left(\left|\lambda_{1}\right|\right) \\
g_{\text {max }_{1}}=32-10 \log (15) \\
g_{\text {max }_{1}}=20,24
\end{gathered}
$$

Reemplazando la ecuación (8) en ecuación (5) se obtiene la ecuación (9).

$$
20,24 *\left(n_{1}-1\right)<S G_{1} \leq 20,24 * n_{1}
$$

Procediendo de manera similar para los demás enlaces de la red. Asimismo, para un enlace de un grupo de estación a una estrella, tendiendo en cuenta que la ganancia del amplificador es de $19,72 \mathrm{~dB}$, como se muestra a continuación en las ecuaciones (10), (11) y (12):

$$
\begin{gathered}
P_{j} \leq P_{\max }-\propto * L_{j}-10 \log \left(D_{j}-1\right)+g_{\max _{j}} * n_{l} \\
P_{1} \leq 3-0,25 * 1-10 \log (16-1)+19,72 * n_{17} \\
P_{1} \leq 3-11,51+19,72 * n_{17}
\end{gathered}
$$

Se procede de manera similar para los demás grupos de estaciones hacia las estrellas. Además, para los enlaces entre una estrella y un grupo de estaciones se tiene las ecuaciones (13), (14) y (15) que:

$$
\begin{gathered}
P_{1} \geq P_{\text {sen }}+\propto * L_{1}-\left(P_{\text {sen }}-10 \log \left(D_{\text {imax }}-1\right)\right) * n_{l} \\
P_{1} \geq-32+0,25 * 1-(32-10 \log (70-1)) * n_{18} \\
P_{1} \geq-32+13,86 n_{18}
\end{gathered}
$$

Luego, se tienen las restricciones de las estrellas, se requiere que la potencia en la salida de cada estrella en cada longitud de onda sea al menos igual a $\mathrm{P}_{\text {sen }}$ como en las ecuaciones (16), (17) y (18).

$$
\begin{gathered}
P_{1} \leq P_{\max }-10 \log \left(D_{1}-1\right)-10 \log \left(\left|\lambda_{\operatorname{lmax} 2}\right|\right) \\
P_{1} \leq 3-10 \log (16-1)-10 \log (55) \\
P_{1} \leq 3-29,16 \leq-26,16
\end{gathered}
$$

De manera similar se hace para las demás estrellas que tiene la red.

El tercer módulo y el cuarto es calcular el número de amplificadores y la ubicación exacta de los mismos, para esto se realizó un programa en Matlab, mostrando el código en un diagrama de flujo en la figura 2 que, al ingresarle los principales datos de la red, que se encuentran en la tabla 2, éste genera como resultado el número de amplificadores que deben haber en cada enlace de la red, incluyendo los del grupo de estaciones, las potencias de cada estrella, la ganancia total del enlace, la ganancia de los amplificadores con las distancias en donde deben estar ubicados y el número total de amplificadores que se encuentran en la red, de esta manera se obtienen los siguientes datos:

$\mathrm{P}_{1}=-26.16 \mathrm{dBm} ; \quad \mathrm{P}_{2}=-23.94 \mathrm{dBm} ;$

$\mathrm{P}_{3}=-22.85 \mathrm{dBm} ; \quad \mathrm{P}_{4}=-22.85 \mathrm{dBm}$

$\mathrm{P}_{5}=-21.31 \mathrm{dBm} ; \quad \mathrm{P}_{6}=-21.77 \mathrm{dBm}$;

$\mathrm{P}_{7}=-22.18 \mathrm{dBm} ; \quad \mathrm{P}_{8}=-22.56 \mathrm{dBm} ;$

$\mathrm{P}_{9}=-22.11 \mathrm{dBm}$;

$\mathrm{n} 1=\mathrm{n} 4=\mathrm{n} 5=\mathrm{n} 6=\mathrm{n} 7=\mathrm{n} 10=\mathrm{n} 11=\mathrm{n} 12=$ $\mathrm{n} 13=2$; 


\section{investigación}

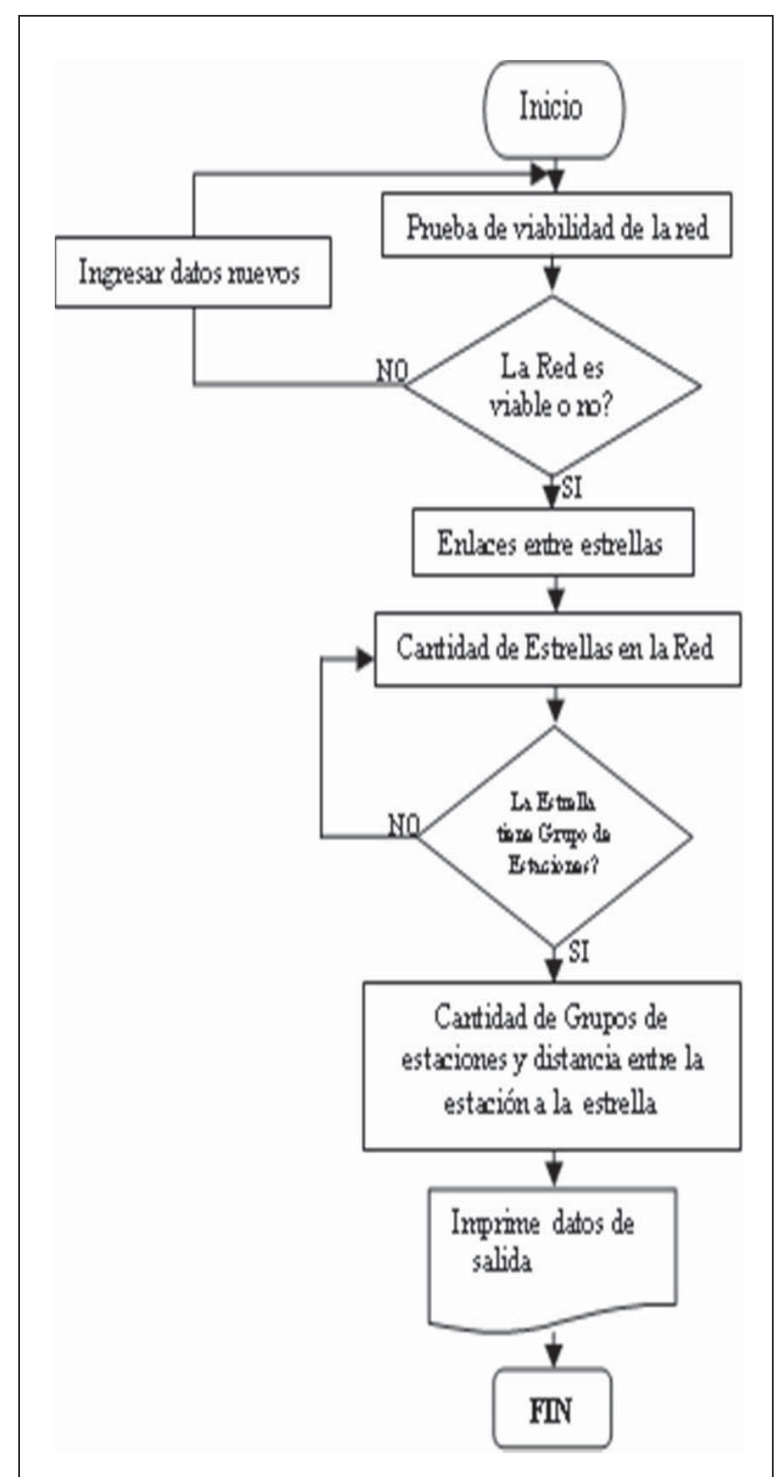

Figura 2. Diagrama de flujo del código de las ecuaciones enteras lineales.

Fuente: elaboración propia

$\mathrm{n} 2=\mathrm{n} 9=\mathrm{n} 15=3$

$\mathrm{n} 3=\mathrm{n} 8=\mathrm{n} 14=\mathrm{n} 16=1$

$\mathrm{n} 17=\mathrm{n} 18=\mathrm{n} 19=\mathrm{n} 20=\mathrm{n} 21=\mathrm{n} 22=\mathrm{n} 23=\mathrm{n} 24=$

$\mathrm{n} 25=\mathrm{n} 26=\mathrm{n} 27=\mathrm{n} 28=\mathrm{n} 29=\mathrm{n} 30=\mathrm{n} 31=\mathrm{n} 32$

$=\mathrm{n} 33=\mathrm{n} 34=0$;

$\mathrm{SG}_{1}=50.43 \mathrm{~dB} \mathrm{SG}_{2}=47.34 \mathrm{Db}$
$\mathrm{SG}_{3}=26.90 \mathrm{~dB} \quad \mathrm{SG}_{4}=24.72 \mathrm{~dB}$

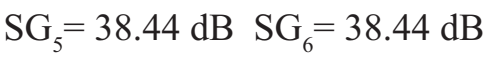

$\mathrm{SG}_{7}=26.12 \mathrm{~dB} \quad \mathrm{SG}_{8}=25.67 \mathrm{~dB}$

$\mathrm{SG}_{9}=41.85 \mathrm{~dB}^{\mathrm{SG}} \mathrm{SG}_{10}=42.76 \mathrm{~dB}$

$\mathrm{SG}_{11}=34.62 \mathrm{~dB} \mathrm{SG}_{12}=35.44 \mathrm{~dB}$

$\mathrm{SG}_{13}=26.00 \mathrm{~dB} \mathrm{SG}_{14}=26.76 \mathrm{~dB}$

$\mathrm{SG}_{15}=35.28 \mathrm{~dB} \mathrm{SG}_{16}=35.19 \mathrm{~dB}$

Dentro del proceso de la prueba de viabilidad se ingresan algunos parámetros técnicos de la red, como son: el mayor grado de la estrella en la red, la mayor cantidad de longitudes de onda en la red, la potencia máxima del amplificador $(\mathrm{dBm})$, el nivel de sensibilidad del amplificador $(\mathrm{dBm})$ y el valor de la atenuación de la fibra óptica $(\mathrm{dB} /$ $\mathrm{Km}$ ), con el fin de ser evaluados por la ecuación que se encuentra en el primer modulo, para poder determinar si la red es viable o no. En caso de que la red no sea viable, es necesario cambiar los parámetros para que sean nuevamente evaluados.

Para los enlaces entre estrellas se requiere de los siguientes parámetros, que son necesarios para evaluar el valor de las potencias, estos son: el número de enlaces que tiene la red, la longitud de onda que lleva el enlace, la distancia que hay entre cada una de las estrellas y el número de las estrellas en las que se encuentra el enlace.

Además, en la cantidad de estrellas en la red se ingresa el número de estrellas que se encuentran en la red, el grado de cada una de las estrellas, la cantidad máxima de longitudes que entran a la estrella, y se realiza la pregunta en referencia a si, en esa estrella, se encuentran grupos de estaciones; si los hay, se ingresan cuántas estaciones hay y la distancia entre la estación y la estrella; 
si por el contrario, no hay grupo de estaciones, se sigue a la siguiente estrella hasta finalizar este proceso. Después, internamente en el programa, se evalúan los valores de las potencia que tiene cada una de las estrellas, la ganancia máxima de los amplificadores, la ganancia total requerida para cada uno de los enlaces, el número de amplificadores en cada enlace y la ubicación de estos.

\subsection{Diseño del algoritmo genético}

El algoritmo genético es una herramienta matemática que se utiliza para resolver problemas asociados a la optimización y a la búsqueda de soluciones óptimas [5]. El propósito de éste es poder hallar el mínimo o máximo de una función objetivo, que para este caso es hallar el mínimo número de amplificadores en la red.

En los algoritmos genéticos se parte de un conjunto de posibles soluciones para un problema dado, al que se le denomina población, y a cada una de las posibles soluciones se les llama individuos [6] y [7]. Por medio de una serie de operadores llamados operadores genéticos, como: selección, cruce y mutación, que se utilizan para hacer evolucionar a los individuos de tal forma que vayan sobreviviendo los mejores.

Como se observa en la figura 3, el AG primero crea una población inicial, dependiendo de estos valores, se hallará el número de los amplificadores en cada enlace y, por consiguiente, la sumatoria de esto para obtener el total de amplificadores en la red.

Es importante tener claro que, para realizar la creación de esta población, es necesario que el algoritmo tenga el rango de búsqueda de cada una de las variables que, en este caso, serán las potencias de cada estrella, es decir, las nueve potencias (P1, P2, P3, P4, P5, P6, P7, P8 y P9), este rango

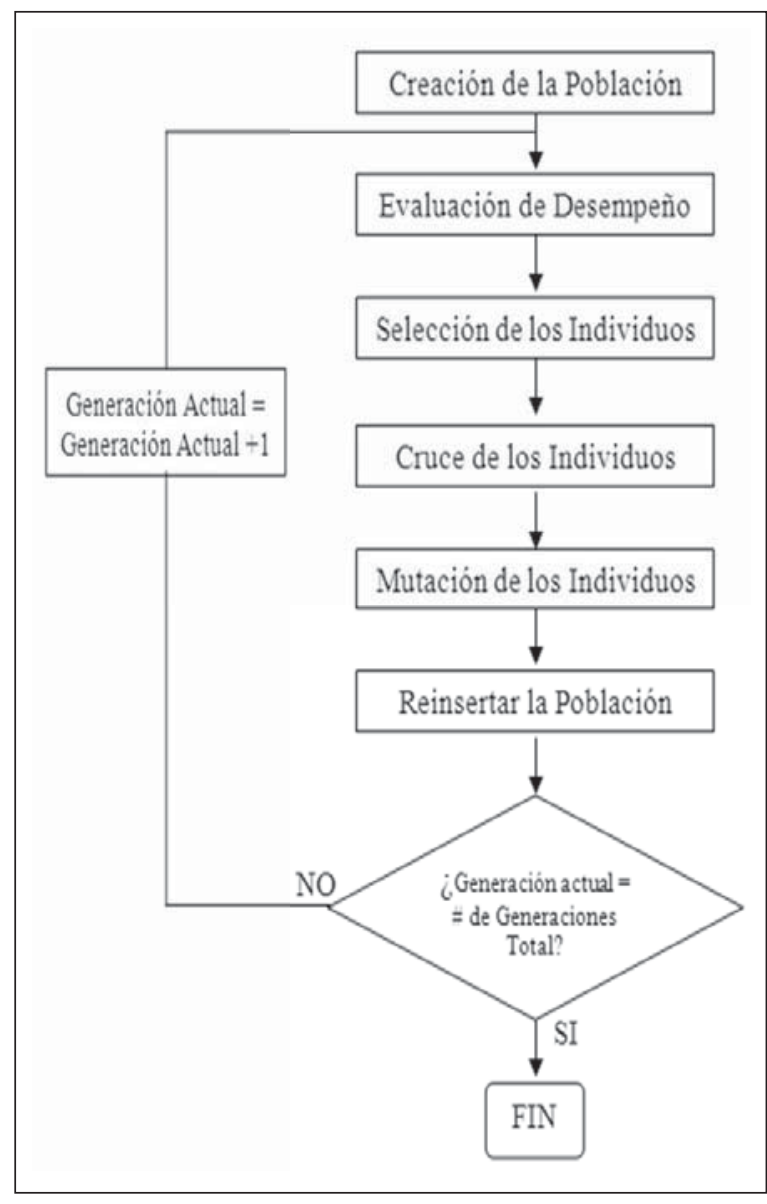

Figura 3. Diagrama de flujo del algoritmo genético. Fuente: elaboración propia

se toma de acuerdo a los valores de las potencias de entrada a los amplificadores.

Después de crear la población inicial, cada potencia tiene valores aleatorios, donde son evaluados por medio de la función objetivo que se obtuvo del despeje de ecuaciones, en donde se realizan las restricciones de los enlaces entre estrellas, grupos de estaciones a las estrellas y viceversa. Gracias a esto, se obtiene un valor del número total de amplificadores en la red, en donde se realiza la evaluación de desempeño, es decir, al menor valor se le da cierta probabilidad de que sobreviva a la siguiente generación, es decir, que sean posiblemente seleccionados 


\section{investigación}

para que puedan realizar el cruce entre estos individuos, el cual determina la evolución de la población, éste escoge dos genes que fueron seleccionados para ser cruzados, que consiste en conseguir una solución local sin llegar aún al resultado óptimo. Los resultados de estos individuos serán posteriormente mutados, donde se modifica aleatoriamente alguno de los genes del individuo descendiente, con el fin de aumentar la diversidad genética que favorece la aparición de individuos con un código genético distinto, con la posibilidad de que sea el mejor.

Por último, se realiza la reinserción de la población, en donde se tiene como resultado el mínimo número de amplificadores. Finalmente, se determina la condición de terminación del algoritmo por medio de dos situaciones: la primera es cuando el algoritmo realiza todas las generaciones preestablecidas; la segunda, es cuando la función fitness logre un valor determinado el cual sea la mejor solución para el problema.

\section{- CÓDIGO DEL ALGORITMO GENÉTICO}

\%\% - - - INICIO DEL AG - - - -

NIND $=40 ; \%$ Número individuos población

MAXGEN = 100; \% Número de generaciones

NVAR $=9 ; \%$ Número de variables

PRECI $=20 ; \%$ Precisión binaria

FieldD $=[\operatorname{rep}([\mathrm{PRECI}],[1, \mathrm{NVAR}]) ; \quad[-20.23 ;-32]$, [-14.59;-32], [-15.46;-32], [-16.55;-32], [-18.02;$32], \quad[-18.98 ;-32], \quad[-20.23 ;-32], \quad[-22 ;-32]$, $[-25.01 ;-32] ; \operatorname{rep}([0 ; 0 ; 1 ; 1],[1, N V A R])]$;

\% Población Inicial

Chrom $=\operatorname{crtbp}($ NIND,NVAR $*$ PRECI);
\% Mejor de la población actual

Best $=[]$;

gen $=1 ; \%$ Contador de generaciones

$\% \%$ Evaluación de la función objetivo

pobred $=$ bs2rv(Chrom,FieldD $)$;

$[\mathrm{N}]=\operatorname{size}($ pobred $)$;

VP1 $=\operatorname{pobred}(:, 1)$;

VP2 $=\operatorname{pobred}(:, 2)$

$\mathrm{VP} 3=\operatorname{pobred}(:, 3)$

$\mathrm{VP} 4=\operatorname{pobred}(:, 4)$

VP5 $=\operatorname{pobred}(:, 5)$

VP6 $=\operatorname{pobred}(:, 6)$;

VP7 $=\operatorname{pobred}(:, 7)$;

$\mathrm{VP} 8=\operatorname{pobred}(:, 8)$

VP9 $=\operatorname{pobred}(:, 9)$;

for a $=1: \mathrm{N} ; \%$ Recorre la población generada por el $\mathrm{AG}$

$\mathrm{P} 1=\mathrm{VP} 1(\mathrm{a}) ; \%$ Envia P1

$\mathrm{P} 2=\mathrm{VP} 2(\mathrm{a}) ; \%$ Envia P2

$\mathrm{P} 3=\mathrm{VP} 3(\mathrm{a}) ; \%$ Envia P3

$\mathrm{P} 4=\mathrm{VP} 4(\mathrm{a}) ; \%$ Envia P4

P5 = VP5(a); \%Envia P5

P6 = VP6(a); \%Envia P6

$\mathrm{P} 7=\mathrm{VP} 7(\mathrm{a}) ; \%$ Envia P7

$\mathrm{P} 8=\mathrm{VP} 8(\mathrm{a}) ; \%$ Envia P8

$\mathrm{P} 9=\mathrm{VP} 9(\mathrm{a}) ; \%$ Envia P9 


\section{investigación}

$\operatorname{objV}(\mathrm{a}, 1)=\operatorname{objfunred1}(\mathrm{P} 1, \mathrm{P} 2, \mathrm{P} 3, \mathrm{P} 4, \mathrm{P} 5, \mathrm{P} 6, \quad[$ minimo,ubi $]=\min (\mathrm{Obj}$ VSel $)$;

P7, P8, P9);

end

$[\operatorname{minimo}, \mathrm{ubi}]=\min (\mathrm{objV})$;

Best $=[$ Best; minimo,

VP1(ubi), VP2(ubi),

VP3(ubi), VP4(ubi),

Best $=[$ Best; minimo, VP1(ubi), VP2(ubi), VP3(ubi), VP4(ubi), VP5(ubi), VP6(ubi), VP7(ubi), VP8(ubi), VP9(ubi)];

\%Matriz que contiene El mínimo de la función objetivo

$\% \%$ Loop generacional

while gen $<$ MAXGEN;

$\%$ Asignación desempeño

VP5(ubi), VP6(ubi),

VP7(ubi), VP8(ubi),

VP9(ubi)];

$\% \%$ Reinsertar descendientes

a población actual

$[$ Chrom objV] $=\operatorname{reins}($ Chrom,

FitnV $=$ ranking(objV);

SelCh, 1, 1, objV, ObjVSel);

\% Selección de individuos

gen $=$ gen +1 ;

SelCh $=\operatorname{select}(\langle$ sus $\rangle$, Chrom,

end

FitnV);

$\% \%$ RECUPERA EL MEJOR VALOR DE LA MATRIZ BEST

$\%$ Cruce

$\mathrm{SelCh}=$ recombine

(〈xovsp〉,SelCh,0.7);

\% Mutación

Best1 = Best(:,1);

$[\mathrm{m}, \mathrm{i}]=\min ($ Best 1$)$;

Numero_Total_de_Amplificadores_en_la_ $\mathrm{RED}=\operatorname{Best}(\mathrm{i}, 1)$

SelCh $=\operatorname{mut}($ SelCh $)$;

$\mathrm{VP} 1=\operatorname{Best}(\mathrm{i}, 2)$

$\%$ Evaluar descendientes

$\mathrm{VP} 2=\operatorname{Best}(\mathrm{i}, 3)$

pobred $=$ bs2rv $(\mathrm{SelCh}$,

VP3 $=$ Best $(\mathrm{i}, 4)$

FieldD);

$\mathrm{VP} 4=\operatorname{Best}(\mathrm{i}, 5)$

$\operatorname{ObjVSel}(\mathrm{a}, 1)=$

VP5 $=$ Best $(\mathrm{i}, 6)$

objfunred1(P1, P2, P3, P4,

VP6 $=$ Best $(i, 7)$

P5, P6, P7, P8, P9);

VP7 $=\operatorname{Best}(\mathrm{i}, 8)$ 
$\mathrm{VP} 8=\operatorname{Best}(\mathrm{i}, 9)$

$\mathrm{VP9}=\operatorname{Best}(\mathrm{i}, 10)$

\section{RESULTADOS}

Tras la ejecución del algoritmo genético, se obtienen los siguientes resultados:

$$
\begin{array}{ll}
\mathrm{A}=28 & \\
\mathrm{P}_{1}=-22.2747 \mathrm{dBm} & \mathrm{P}_{2}=-21.2470 \mathrm{dBm} \\
\mathrm{P}_{3}=-18.0261 \mathrm{dBm} & \mathrm{P}_{4}=-19.5547 \mathrm{dBm} \\
\mathrm{P}_{5}=-17.5332 \mathrm{dBm} & \mathrm{P}_{6}=-22.4750 \mathrm{dBm} \\
\mathrm{P}_{7}=-24.1090 \mathrm{dBm} & \mathrm{P}_{8}=-29.4567 \mathrm{dBm} \\
\mathrm{P}_{9}=-31.3371 \mathrm{dBm} & \\
\mathrm{n}_{1}=\mathrm{n}_{3}=\mathrm{n}_{5}=\mathrm{n}_{6}=\mathrm{n}_{7}=\mathrm{n}_{9}=\mathrm{n}_{10}=\mathrm{n}_{11}=\mathrm{n}_{12}=\mathrm{n}_{15} \\
=2 ; \\
\mathrm{n}_{2}=3 ; \\
\mathrm{n}_{4}=\mathrm{n}_{8}=\mathrm{n}_{13}=\mathrm{n}_{14}=\mathrm{n}_{16}=1 ; \\
\mathrm{n}_{17}=\mathrm{n}_{18}=\mathrm{n}_{19}=\mathrm{n}_{20}=\mathrm{n}_{21}=\mathrm{n}_{22}=\mathrm{n}_{23}=\mathrm{n}_{24}=\mathrm{n}_{25}=\mathrm{n}_{26} \\
=\mathrm{n}_{27}=\mathrm{n}_{28}=\mathrm{n}_{29}=\mathrm{n}_{30}=\mathrm{n}_{31}=\mathrm{n}_{32}=\mathrm{n}_{33}=\mathrm{n}_{34}=0 ; \\
\mathrm{SG}_{1}=49.2377 \mathrm{~dB} & \mathrm{SG}_{2}=48.5323 \mathrm{~dB} \\
\mathrm{SG}_{3}=29.0309 \mathrm{~dB} & \mathrm{SG}_{4}=22.5891 \mathrm{~dB} \\
\mathrm{SG}_{5}=36.9114 \mathrm{~dB} & \mathrm{SG}_{6}=39.9686 \mathrm{~dB} \\
\mathrm{SG}_{7}=29.2315 \mathrm{~dB} & \mathrm{SG}_{8}=22.5585 \mathrm{~dB} \\
\mathrm{SG}_{9}=37.3582 \mathrm{~dB} & \mathrm{SG}_{10}=47.2418 \mathrm{~dB} \\
\mathrm{SG}_{11}=33.3960 \mathrm{~dB} & \mathrm{SG}_{12}=36.6640 \mathrm{~dB} \\
\mathrm{SG}_{13}=21.0323 \mathrm{~dB} & \mathrm{SG}_{14}=31.7277 \mathrm{~dB} \\
\mathrm{SG}_{15}=33.7496 \mathrm{~dB} & \mathrm{SG}_{16}=36.7204 \mathrm{~dB}
\end{array}
$$

Teniendo cuenta que con el algoritmo de ecuaciones enteras lineales se obtenía un número total de amplificadores de 31, el AG permite disminuir este valor en tres amplificadores menos, lo que quiere decir que es la mejor técnica para la optimización del número de amplificadores pues el objetivo principal de este diseño es la disminución del número de amplificadores en la red y, por consiguiente, se va a tener una reducción en los costos de la red; también se obtuvieron otros resultados como las potencias para cada una de las estrellas, la ganancia que tienen los amplificadores, la ganancia total requerida para cada uno de los enlaces, el número individual de amplificadores para cada enlace y la ubicación exacta para cada amplificador.

\subsection{Simulación de la red}

En la simulación se utilizó el software OptiSystem que permite la simulación de sistemas de comunicaciones por fibra óptica en el nivel 1 de la capa OSI, y además contiene una amplia librería de componentes activos y pasivos.

En la figura 4 se muestra el circuito del enlace 1 y se utiliza la topología punto a punto, se hace de esta manera porque en el simulador no se encuentra un demultiplexor bidireccional y un componente que permitan la conversión de óptico a eléctrico. El enlace está conformado por 15 longitudes de onda las cuales van a ser transmitidas por el trasmisor WDM, éste utiliza una modulación de NRZ (No Retorno a Cero) y RZ (Retorno a Cero), se trabajó con NRZ porque, al compararla con la modulación RZ, se observó que ésta mejoraba la señal en el receptor. A continuación sigue el multiplexor WDM, el cual envía las señales que recibió del transmisor para luego enviarlas por la fibra; después, estas señales llegan al amplificador, en este caso se van a tener dos amplificadores EDFA cada uno con la ganancia que generó el algoritmo genético y las distancia en donde deben ser ubicados, después de ser amplificadas las longitudes de onda, estas llegan al demultiplexor WDM, en donde éste las descompone y las convierte a su 


\section{investigación}

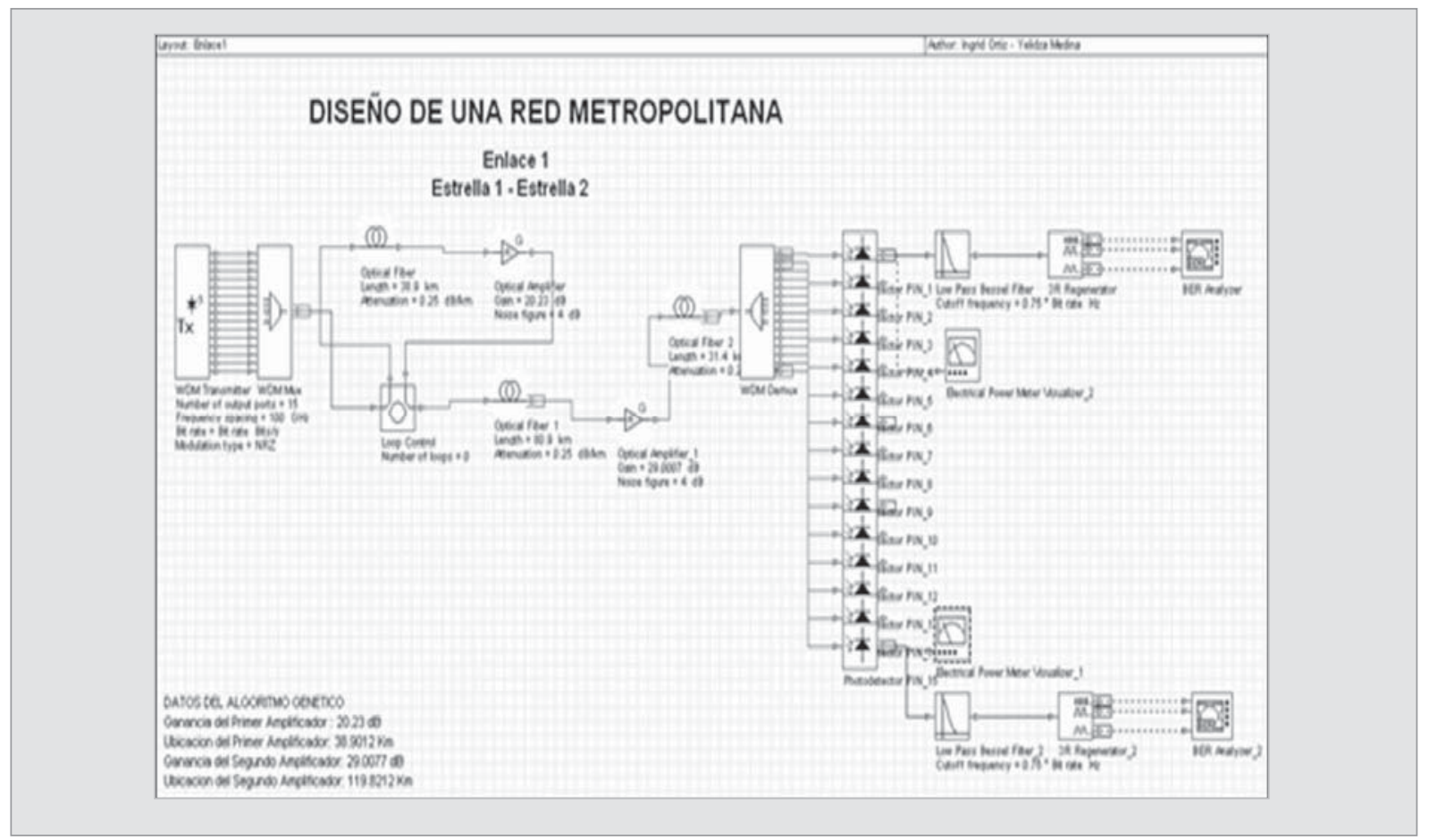

Figura 4. Circuito del enlace 1.

Fuente: elaboración propia

forma original transmitiéndolas a los fotodetectores PIN. Este circuito va a ser el mismo para las ecuaciones enteras lineales, la diferencia entre estos dos son las distancias que van a tener cada uno de los amplificadores y las ganancias para cada uno de estos, por lo tanto, los resultados van a ser mostrados mediante diagrama de ojo y los niveles de potencias para cada uno de estos algoritmos tener los resultados, los cuales se van a mostrar mediante el diagrama de ojo y los niveles de potencia para cada uno de ellos, los cuales se mostraran en las figuras 5 y 6 .

En la figura 5 y la figura 6, se nota una diferencia en el nivel de potencia en las salidas de los receptores, ya que los parámetros de estos dos algoritmos tienen diferentes distancias en donde se ubican los amplificadores, además de las ganancias de cada uno de ellos, es por esto que al comparar estos dos algoritmos se puede observar que el algoritmo genético, además de optimizar el número de amplificadores, permite mejorar el nivel de atenuación, ya que es menor al resultado de la simulación con las ecuaciones enteras lineales.

Además, se observa que el diagrama de ojo, para la figura 5, su abertura tiene un ancho de $0,86 \mathrm{y}$ un alto de 0,71 con un valor mínimo de BER de $8.07 * 10^{-4}$, en donde se puede determinar que la señal de salida sufre una atenuación y tiene interferencia entre símbolos significativa, esto se debe a que el valor de BER no es igual al ideal el cual esta dentro de un rango de $10^{-7}$ a $10^{-12}$. Por consiguiente, en la figura 6 se observa que el diagrama de ojo para éste tiene un valor mínimo de VER, el cual es de $4.8^{*} 10^{-4} \mathrm{y}$ un ancho de 0.89 con un alto de 0.81 , al comparar con el diagrama de ojo de la figura 5 se puede notar que el BER es menor con el algoritmo genético, por lo tanto, el ojo va a tener una mayor abertura, el cual indica que va atener una menor atenuación y la señal va hacer detectada por el receptor coherentemente. 


\section{investigación}

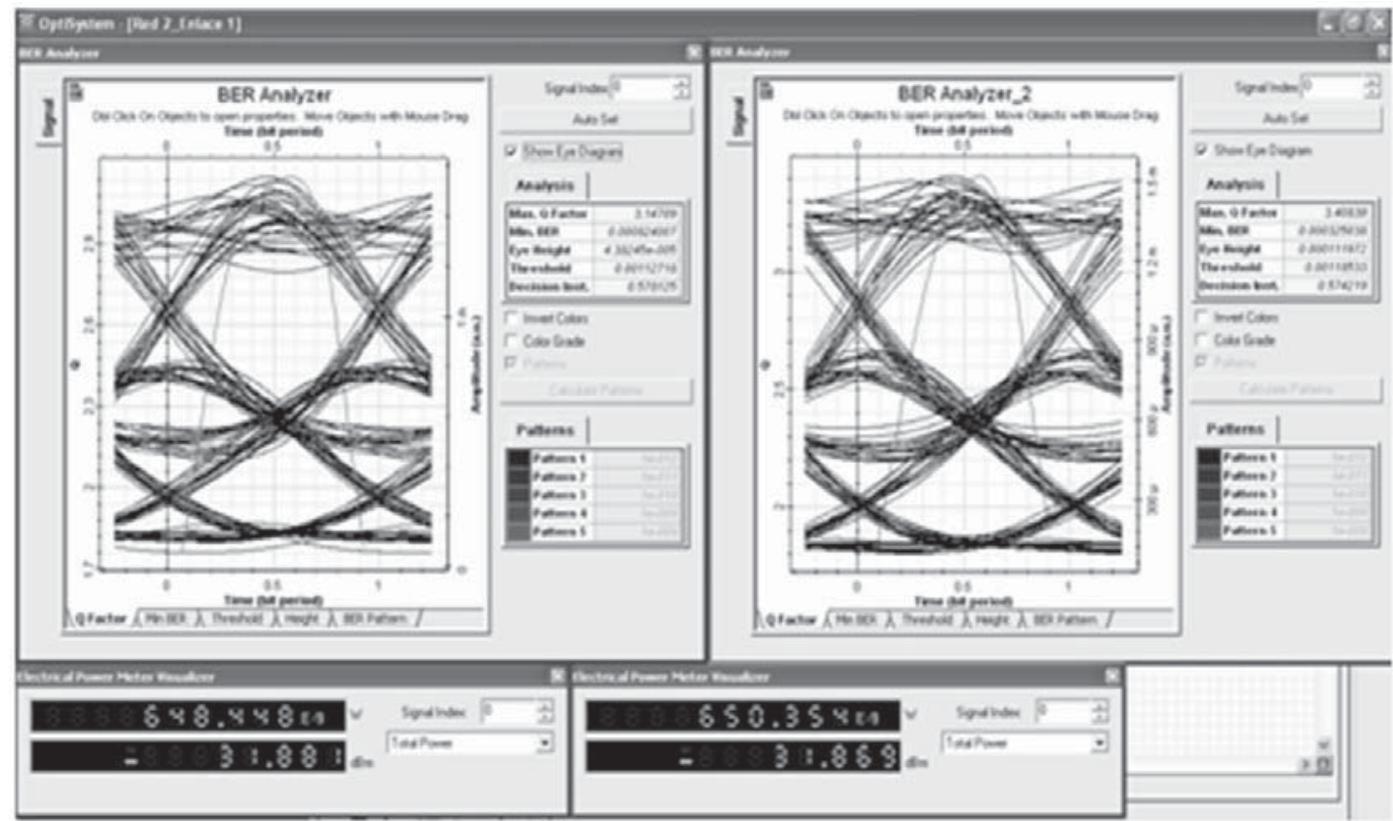

Figura 5. Diagrama de ojo del Enlace 1 con las ecuaciones enteras lineales en las salidas 1 y 15.

Fuente: elaboración propia

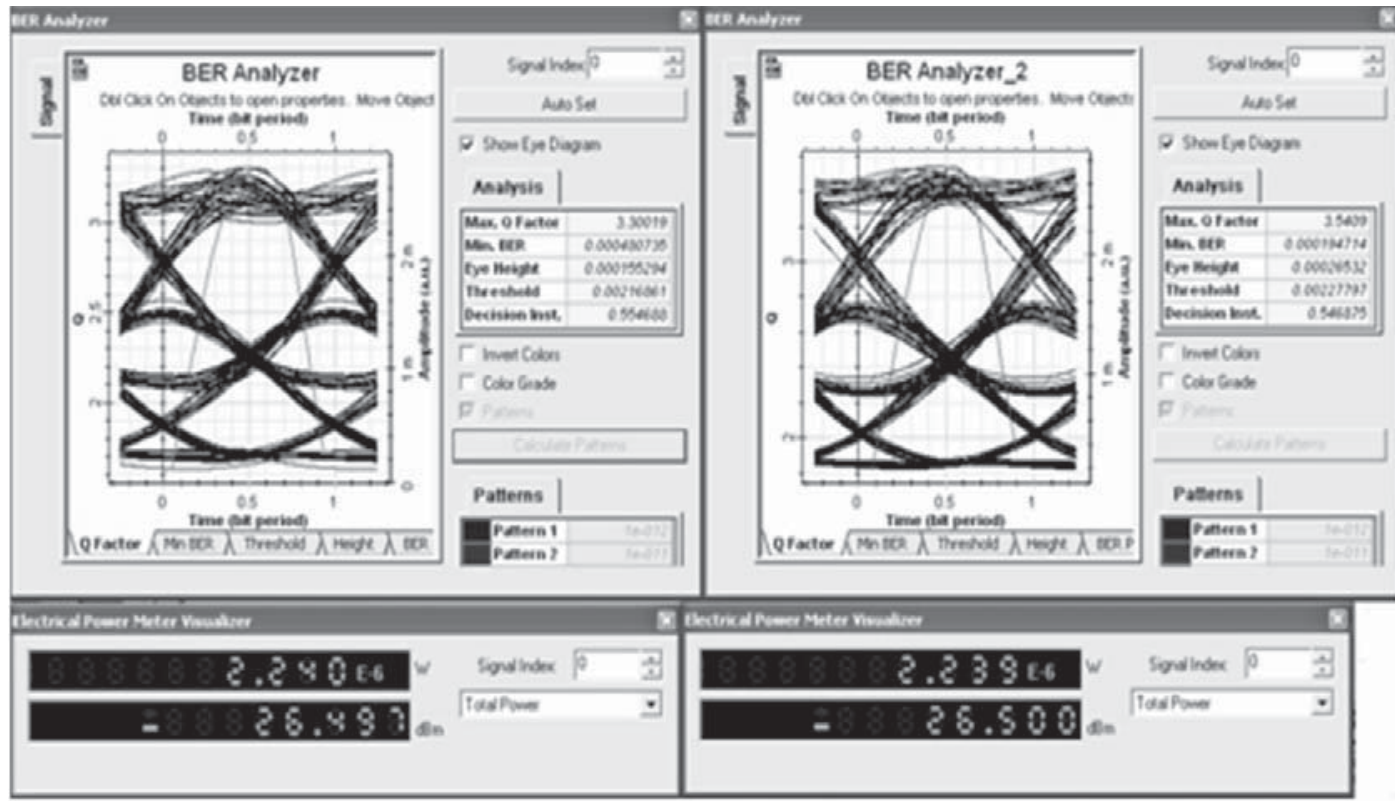

Figura 6. Diagrama de ojo del Enlace 1 con el Algoritmo Genético en las salidas 1 y 15.

Fuente: elaboración propia 


\section{investigación}

\section{CONCLUSIONES}

Las redes ópticas tienen una desventaja en cuanto a sus componentes, los cuales comercialmente son muy costosos, específicamente los amplificadores ópticos, cuyo valor oscila entre 20.000 a 60.000 euros, esto indica que si se pone más de un amplificador en la red incrementaría los costos de esta. Para evitar esto se buscó una técnica de optimización la cual minimizara el número de amplificadores requeridos por la red. De esta manera, se utilizaron dos algoritmos que se trabajaron en Matlab, el primer algoritmo se realizó por medio de las ecuaciones enteras lineales, el cual dio como resultado 31 amplificadores. Mientras que el algoritmo genético dio como resultado 28 amplificadores en la red, lo que quiere decir que con los algoritmos genéticos se obtuvo una disminución del $10 \%$ en el número de amplificadores que se van a requerir en la red.

Teniendo en cuenta la información adquirida sobre algoritmos genéticos y por pruebas realizadas, se puede decir que entre más grande sea la po- blación inicial y más generaciones se encuentren en este, es posible obtener un mejor resultado en el desempeño de los individuos, aproximándolos a una mejor evaluación en la función de aptitud, debido a que esta escoge los mejores resultados entre los individuos, haciendo que sobrevivan a la siguiente generación.

Por medio del software OptiSystem, se realizaron las simulaciones de cada uno de los enlaces, se utilizó la topología punto a punto ya que en el simulador no hay un componente que permita convertir la señal óptica a eléctrica o viceversa. Al evaluar cada uno de estos enlaces se obtuvo como resultado el nivel de potencia en los receptores y el diagrama de ojo. Por medio de éste, se pudo evaluar cada enlace, permitiendo observar por medio gráfico las atenuaciones y la interferencia entre símbolos que se encuentran en la señal de salida en los receptores, para determinar si éste tenía la capacidad de detectar la señal transmitida o si, por el contrario, no tendría la capacidad de discriminar nada, en este caso la tasa de errores en la transmisión seria elevadísima.

\section{REFERENCIAS}

[1] B. Ramamurthy, Design of optical WDM networks LAN MAN and WAM architectures. United States of America: Kluwer Academic Publishers,,2001.

[2] M. Borellas, Optical Component for WDM Light Wave Networks, Qluwer Academic Publishers, 2001.

[3] P. Ramos, Redes ópticas DWDM (Dense Wavelength Division Multiplexing), [en linea]. Disponible: http://www.radioptica. com/Fibra/dwdm.asp

[4] A. Keyser, Optical Fiber Comunication, McGraw Hill, 2000.
[5] D.Goldberg, Genetic Algorithms in search, optimization, and machine learning, $\mathrm{Ed}$ Addison-Wesley, 2000.

[6] I. Ruge, "Diseño de un regulador adaptable basado en algoritmos genéticos", Revista Colombiana de Tecnologías de Avanzada, Vol. 2, No.6, pp. 13-19, 2005.

[7] I. Ruge, y M. Alvis, "Aplicación de los algoritmos genéticos para el diseño de un controlador PID adaptativo", Revista Tecnura Universidad Distrital Francisco José de Caldas, Vol. 25, pp. 83-90, 2009. 